\title{
Quality Control of Platelets Concentrates; an In Vitro Fate Prediction Model System of PCs Transfusion
}

\section{Timori $\mathrm{NH}^{1}$ and Badlou $\mathrm{BA}^{2^{*}}$}

${ }^{1}$ Blood Transfusion Research Center, High Institute for Research and Education in Transfusion Medicine, Tehran, Iran

${ }^{2}$ Department of Research and Development, BBAdvies and Research, Zeist, The Netherlands

"Corresponding author: Bahram Alamdary Badlou (CEO), Hematology/Transfusion Medicine-Platelets, BBAdvies and Research, Department of Research and Development, 3706 XA, Zeist, The Netherlands, Tel: +31638628933; Fax: +31302211328; E-mail: bbadlou@casema.nl

Received date: Apr 10, 2015, Accepted date: May 11, 2015, Publication date: May 18, 2015

Copyright: $\odot 2015$ Timori $\mathrm{NH}$, et al. This is an open-access article distributed under the terms of the Creative Commons Attribution License, which permits unrestricted use, distribution, and reproduction in any medium, provided the original author and source are credited.

\section{Abstract}

Introduction: We previously described that metabolic resting preserves old and cold platelets (PLTs) concentrates (PCs). In Badlou et al. 2006 we have established that P-selectin involved as an intermediate in binding, and the combination of PS exposure with (-out) changes in GPIb in phagocytosis of PCs. Using a standard threshold over the interaction between donors PLTs and patient's phagocytes might increase success of transfusion, and decrease side effects, pretransfusion.

The aim of this study is to introduce a novel method, which could be used as an in-vitro prediction model system to envisage PCs fate, posttransfusion.

Materials and Methods: Human PCs were stored for different periods to obtain a wide range of surface expressed P-selectin and-PS exposure. The quantitative change in GPIb was deduced from decreased binding of an anti GPIb- antibody measured by FACS flowcytometry. Correlations established in RT stored-PCs were compared with PCs stored at $0^{\circ} \mathrm{C}$, and metabolic suppressed stored at $4^{\circ} \mathrm{C}$ (MSP4).

Results: Qualitative and quantitative analysis revealed that 'good PCs' which had low P-selectin and PS exposure, and high GPIb expression were not removed by phagopcytes. Comparison studies between $\mathrm{C} 22, \mathrm{C} 0$ and MSP4 showed that at a given amount of PS exposure and GPIb expression, phagocytes immediately removed 'bad PCs'. Threshold- dependent removal might be caused by ageing-dependent lesions. At high GPIb expression was phagocytosis almost $0 \%$, when GPIb expression decreased below certain degree phagocytosis increased, remarkably.

Discussions: Our results signify that these chosen three markers combination measurements are reliable markers for phagocytic (ir-) responsiveness. In the near future, when these thresholds established as standard thresholds then any (Para-)medic can predict on the basis of relatively simple analysis, whether delivered PCs from the blood banks are immunogenic or not. In conclusion, our introduced in-vitro model system could be used as the quality control of any randomly selected and prepared PCs, pretransfusion.

Keywords: Transfusion; Platelets; Human; Prediction model system; In vitro; Immune response

\section{Introduction}

We previously described that metabolic resting preserves better PCs quality and quantity old and cold platelet (PLT) concentrates (PCs) [1]. Moreover, Badlou et al. showed that P-selectin acts as an intermediate marker in binding and the different combinations of PS exposure with (out) conformational changes in GPIba [2] as intermediates in phagocytosis [3].

Using an in vitro assay for pre-analysis of the interaction between PLTs and macrophages might enhance success of transfusion and decreases side effects significantly. There is little insight in the relative contribution of these regulators of PLT destruction by macrophages in different patients.
There are raising evidences that in the field of PCs transfusion optimal PLT storage in vitro correlates directly with optimal hemostatic effectiveness and survival in vivo [4,5].

Moreover, optimal preservation of PLT reactivity results in better PLT functions [2,6,7]. Nevertheless, there is a need for PLT prediction assay that under laboratory conditions is able to predict how PCs behave themselves in the circulating blood of any random patient.

Different markers have been tested as indicators of the so called platelet storage lesion (PSL), such as P-selectin expression (PSE) $[7,8]$, CD40/CD40 ligand $[9,10]$ and glycoprotein (GP) IIbIIIa [11], changes in mean platelet volume (MPV), sphere-to-disc changes [12,13], actin assembly [14,15], serotonin release [16,17], changes in GPIb-V-IX and GPVI expressions [18,19], PS exposure [20,21], loss of swirling [22], osmotic pressure response [23], and changes in PLTs count caused by agglutination [24].

Although each marker provides insight in the activation state of the PLTs, none of them is a perfect predictor of the quality of transfused 
PLTs. An additional problem is the lack of comparative studies with data on sensitivity and specificity of the different markers.

Prolonged storage induces a gradual increase in PSE [25-27], PS exposure [3,21], and a decrease in GPIba expression [28-30]. Leytin et al. described that PSE showed a positive and GPIb expression a negative correlation with phagocytosis of PLTs in vivo [8].

Previously we provided evidence that binding of PLTs to macrophages is mediated by surface expressed P-selectin and phagocytosis by exposed PS and changes in GPIb. Changes in GPIb were detected as a decrease in the binding of a PE-labeled Moab against AA1-35on the $\mathrm{N}$-terminal flank and thought to be related to the clustering of GPIb observed after cooling of PLTs [31].

The aim of this study is to introduce a novel in-vitro prediction model system to predict PCs fate, posttransfusion. We hypothesis that using changes in these three PLTs markers (P-selectin, PS exposure, and GPIb), would be enough to (dis-)qualify any randomly selected and prepared PCs, pretransfusion.

\section{Materials and Method}

This retrospective study of data were prepared and analyzed from 30 different independent human voluntary donors with inform consent as previously described [3]. The quantitative and qualitative binding and phagocytosis assays were done with BD-FACSCalibur flow cytometry, and high confocal (video) microscopic photos acquired under flow, and were measured as described previously $[3,30]$. The data were analyzed using WinMDI-and Graphpad 6.0 softwares and statistics. The data presented are Mean \pm SEM of 30 randomly selected assays with human isolated PCs in-vitro.

\section{Results}

Freshly isolated resting PCs (Figure 1) stored at room temperature for 1 hour showed a single population with high affinity to the GPIb (Figure 2). Retrospective study of interaction data acquired from human PCs and PMA stimulated THP-1 cells under ex-vivo condition revealed that less than $5 \%$ of fresh PLTs were bound and removed by THP1 phagocytes.

We have chosen this threshold and cut-off limit to build and introduce our novel prediction model system. Moreover, our hypothesis was that it should be possible to assess a reconsider quality control of any random PCs, pretransfusion.

Prolonged storage condition was accompanied by an increase in PLTs binding to and phagocytosis by macrophages in a stepwise manner (Figure 2A and 2B).

In freshly isolated PCs the P-selectin expression and binding were less than $5 \%$ but after prolonged storage condition increased binding to macrophages to $25 \pm 15 \%$ while the removal percentage of PLTs by macrophages was less than $<5 \%$.

After 48 hrs storage this fraction decreased to $60 \%$ resulting in a step by step increasing of PCs by phagocytes (Figure 2C).

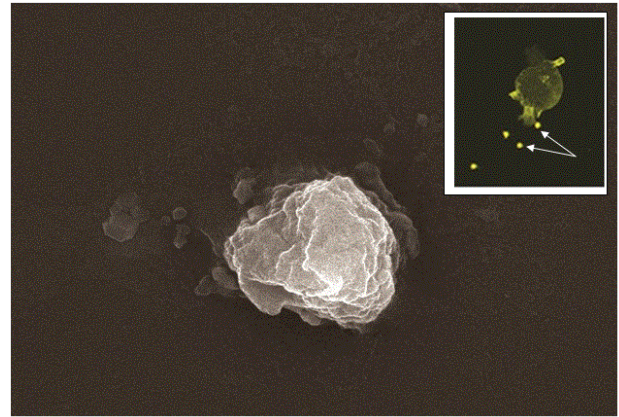

Figure 1: Confocal high resolution microscopy of human platelets in resting state. Insertion. In this video microscopy photo under flow, the PCs labeled with mepacrine (Quinacrine dihydrochloride, Sigma-Aldrisch, Germany) and introduced to the PMA-stimulated THP-1 (un-)differentiated monocytes under ex-vivo condition. These photos give an idea about evidences in relation to the resting PCs, which do not bind and phagocyted by phagocytes $[3,30]$.

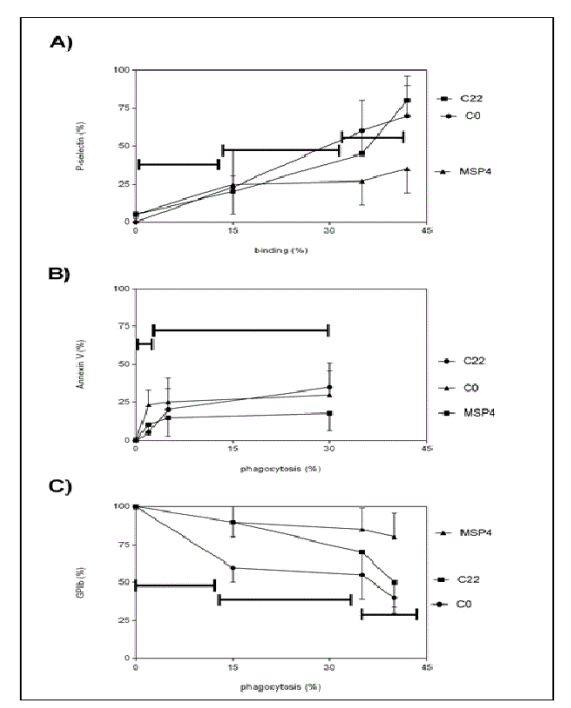

Figure 2: Causal relation between storage temperature, metabolic condition of platelets concentrates versus increasing in phagocytosis. Correlations between A) P-selectin expression vs binding percentage of PCs B) Annexin V binding to Phosphatidyl serine, and C) GPIb expression vs an increase in phagocytosis percentage in platelets from the same donor stored at room temperature $(\mathrm{C} 22)$, ice $0^{\circ} \mathrm{C}(\mathrm{C} 0)$ and in refrigerator $4^{\circ} \mathrm{C}$ after metabolic suppression (MSP4) after $0,40 \mathrm{~min}, 48 \mathrm{~h}$ and $72 \mathrm{~h}$ storage. These figures indicates that the fresh PCs do not bind and phagocyted by macrophages but after prolonged storage step by step, time dependent, and receptors-condition dependently phagocyted by THP-1 macrophages in vitro. Hence, changes in Pselectin expression, PS exposure, and GPIb could be used as a qualification threshold to (dis-) qualify any random PCs prepared by any kind of method.

Fresh PLTs showed a mean \pm SEM about $10 \pm 8 \%$ P-selectin expression in the absence of PMA and $50 \%$ in its presence, confirming 
the role of PMA as a secretion-inducing agent. Phagocytosis of the same platelets was $<5 \%$ with and without PMA removal. After 48 hours storage, there were about $50 \%$ P-selectin expression without PMA and 60\% with PMA. These PLTs showed about 20 and 35\% phagocytosis. Our new acquired data are in line with the concept that P-selectin is an intermediate in binding and bringing PCs on phagocytosis.

We next retrospectively studied the correlations between GPIb peak-2 expression versus phagocytosis, as described [30].

Compared to fresh (T0, assumed as 100\%) the GPIb expression and conformational change showed a threshold of less than 25\% (Figure $2 \mathrm{C}$ ), below which phagocytosis was less than $<6 \%$, but after prolonged storage GPIb expression was decreased and GPIb peak-2 increased in an ageing-dependent manner from 30 up to $90 \%$ which correlated linearly with higher phagocytosis (Figure 3).

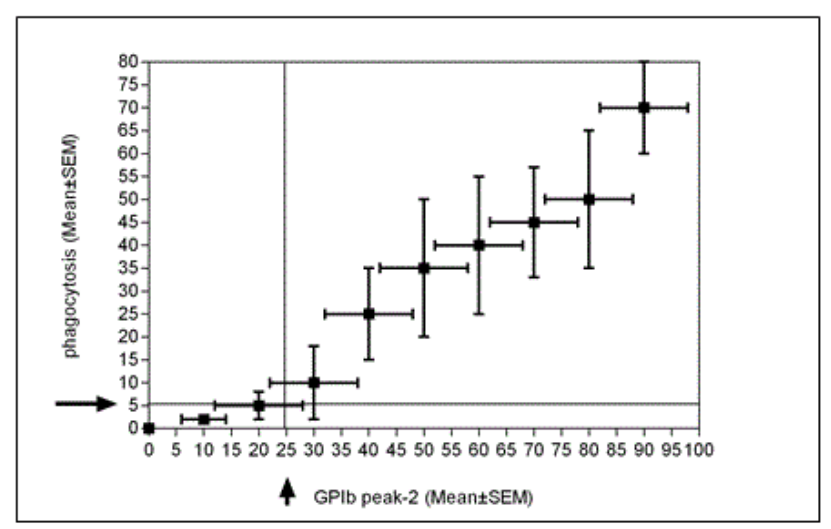

Figure 3: GPIb clustering-like appearance (GPIb peak 2) versus aggravation of phagocytosis of C22 platelets after $0,40 \mathrm{~min}, 48 \mathrm{~h}$ and $72 \mathrm{~h}$ storage. The data presented are the Mean \pm SEM of 20 different experiments in vitro. This figure illustrates the PCs based on quantitative GPIb-decay (-dependent) phagocyted by macrophages with a threshold of $25 \%(n=30)$. Hence, GPIb degradation and changes could be used as biomarker of the recipient's immune response.

Correlations between either P-selectin expression versus binding percentage of PCs; and Annexin V binding to phosphatidyl serine (PS), or GPIb expression versus an increase in phagocytosis percentage indicated that the fresh PCs do not bind and phagocyted by macrophages. Therefore, immune response should be minuscule.

After prolonged storage and ageing-related membrane lesions step by step, time dependent, and receptors-condition dependently the PCs removal increased. Consequently, any significant changes in P-selectin expression, PS exposure, and GPIb could be used as a qualification threshold to (dis-) qualify any random PCs prepared by any kind of method, pretransfusion.

\section{Discussion}

Different extra- and intracellular PLTs markers have been tested as indicators for PCs quality and quantity. PLTs in rest were not removed by phagocytes, which their external and internal proteins are suppressed to remain in their places. In this retrospective study we observed that a slight degree of PS exposure $(<5 \%)$ and GPIb change $(<20 \%)$ do not induce phagocytosis, remarkably. There appears to be a threshold that primes phagocytes to bind and remove PLTs. Moreover only when shape and surface changes exceed this threshold level, PLTs become 'stepwise' targets for destruction and phagocytosis. It is noteworthy that phagocytosis of a fraction of PLTs does not mean whole population would be destroyed subsequently. Any PLT that remain intact and kept all ex- and internal proteins in their position will not be phagocyte as we observed after recovery of metabolic suppressed PLTs (MSP4/48 hrs) [3,30]. Beside we observed that fresh PLTs show a slight degree of P-selectin expression, PS exposure and GPIb change but they were not phagocytized $[3,30]$. We found a marginal threshold for PSE, and even in fresh PCs a slight increase in PSE induced binding to macrophages but finally did not turn over to removal of fresh PCs, however. In old PCs only metabolic recovered PCs has lower phagocytosis which indicates GPIb changes and PS exposure are not irreversible processes and could be repaired metabolically pretransfusion [3,30,32-35]. Interestingly, metabolic suppression prior to cold storage greatly suppressed the expression of phagocytic signals. The relation between PS exposure and GPIb change versus phagocytosis appears to follow the correlation established with RT stored but the low PS expression and GPIb change hamper reliable calculations.

The finding that metabolic suppression also reduces the change in GPIb receptor during prolonged storage is still unexpected phenomenon. GPIb is the receptor that mediates platelet adhesion to VWF [6]. The cooling of PLTs is thought to trigger irreversible GPIb clustering which provides a signal for rapid destruction by phagocytes, confirmed Hoffmeister et al. [35-37] In contrary, also storage at RT $\left(18-24^{\circ} \mathrm{C}\right)$ shows also old PCs more prone to phagocytosis, suggesting that the same mechanism might control the removal of RT-stored PCs, eventually.

Taken together, an slight increase $(<0.5 \%)$ in the PSE, PS exposure and GPIb changes induced more binding to phagocytes but did not further result in clearance of PCs. In contrast, higher than 30\% PS exposure and GPIb change showed direct stepwise increase in phagocytosis with a certain threshold. If the regulation of PLT binding and phagocytosis by macrophages are restricted to surface expression of P-selectin, PS, and changes on GPIb, One might expect that correlations established here might valid for any random PCs stored under different conditions.

It is clear that the present correlations between surface markers for PCs destruction by macrophages are based on an in vitro system with matured THP-1 cells. At present it is uncertain to which extent this set up is an accurate reflection of the destruction of PCs under in-vivo conditions. If such a correlation could be established in future studies, the sensitivity of transfused PCs to be destructed by macrophages could be predicted on the basis a few, rather simple quantitative and qualitative assay(s)-analysis. In the near future, if by any company, pharmaceutical drugs developed and introduced, which can prevent PCs damages and/or restore stored PCs lesion pretransfusion, all can be qualitatively controlled by such simple combination assay.

In conclusion, our results signify that the chosen three markers combination measurement are representative for phagocytic (ir-) responsiveness. These parameters measured in-vitro could be used as the quality control markers of any randomly selected and prepared PCs, pretransfusion. In the near future, when these thresholds established as standard thresholds then any (Para-)medic can predict 
on the basis of relatively simple analysis, whether delivered PCs from the blood banks are immunogenic or not.

\section{References}

1. Naghadeh HT, Badlou BA, Ferizhandy AS, Mohammadreza TS, Shahram $\mathrm{V}$ (2013) Six hours of resting platelet concentrates stored at $22-24{ }^{\circ} \mathrm{C}$ for 48 hours in permeable bags preserved $\mathrm{pH}$, swirling and lactate dehydrogenase better and caused less platelet activation. Blood Transfus 11: 400-404.

2. Gutensohn K, Geidel K, Brockmann M, Siemensen M, Krueger W, et al (2002) Binding of activated platelets to WBCs in vivo after transfusion. Transfusion 42: 1373-1380.

3. Badlou BA, Wu YP, Smid WM, Akkerman JW (2006) Platelet binding and phagocytosis by macrophages. Transfusion 46: 1432-1443.

4. Holme S, Sweeney JD, Sawyer S, Elfath MD (1997) The expression of pselectin during collection, processing, and storage of platelet concentrates: relationship to loss of in vivo viability. Transfusion 37: 12-17.

5. Gurney D, Lip GY, Blann AD (2002) A reliable plasma marker of platelet activation: does it exist? Am J Hematol 70: 139-144.

6. Stepanian A, Ribba AS, Lavergne JM, Fressinaud E, Juhan-Vague I, et al. (2003) A new mutation, S1285F, within the A1 loop of von Willebrand factor induces a conformational change in A1 loop with abnormal binding to platelet GPIb and botrocetin causing type $2 \mathrm{M}$ von Willebrand disease. Br J Haematol 120: 643-651.

7. Leytin V, Mody M, Semple JW, Garvey B, Freedman J (2000) Quantification of platelet activation status by analyzing P-selectin expression. Biochem Biophys Res Commun 273: 565-570.

8. Leytin V, Mody M, Semple JW, Garvey B, Freedman J (2000) Flow cytometric parameters for characterizing platelet activation by measuring P-selectin (CD62) expression: theoretical consideration and evaluation in thrombin-treated platelet populations. Biochem Biophys Res Commun 269: 85-90.

9. Aloui C, Prigent A, Sut C, Tariket S, Hamzeh-Cognasse H, et al. (2014) The signaling role of CD40 ligand in platelet biology and in platelet component transfusion. Int J Mol Sci 15: 22342-22364.

10. Sahler J, Spinelli S, Phipps R, Blumberg N (2012) CD40 ligand (CD154) involvement in platelet transfusion reactions. Transfus Clin Biol 19 98-103.

11. Buensuceso C, de Virgilio M, Shattil SJ (2003) Detection of integrin alpha IIbbeta 3 clustering in living cells. J Biol Chem 278: 15217-15224.

12. Leven RM, Gonnella PA, Reeber MJ, Nachmias VT (1983) Platelet shape change and cytoskeletal assembly: effects of $\mathrm{pH}$ and monovalent cation ionophores. Thromb Haemost 49: 230-234.

13. Kunicki TJ, Tuccelli M, Becker GA, Aster RH (1975) A study of variables affecting the quality of platelets stored at "room temperature". Transfusion 15: 414-421.

14. Mohri H, Asakura Y, Fukushima J, Kawamoto S, Okubo T, et al. (1997) Synthetic peptide from the V3 loop consensus motif with a potent antiHIV activity inhibits ristocetin-mediated vWF-GPIb interaction. Peptides 18: 1289-1293.

15. Hartwig JH, Barkalow K, Azim A, Italiano J (1999) The elegant platelet: signals controlling actin assembly. Thromb Haemost 82: 392-398.

16. Maurer-Spurej E, Pfeiler G, Maurer N, Lindner H, Glatter O, et al. (2001) Room temperature activates human blood platelets. Lab Invest 81: 581-592.

17. de Korte D, Gouwerok CW, Fijnheer R, Pietersz RN, Roos D (1990) Depletion of dense granule nucleotides during storage of human platelets. Thromb Haemost 63: 275-278.

18. Bergmeier W, Rabie T, Strehl A, Piffath CL, Prostredna M, et al. (2004) GPVI down-regulation in murine platelets through metalloproteinasedependent shedding. Thromb Haemost 91: 951-958.
19. Alberio L, Friese P, Clemetson KJ, Dale GL (2002) Collagen response and glycoprotein VI function decline progressively as canine platelets age in vivo. Thromb Haemost 88: 510-516.

20. Furihata K, Clemetson KJ, Deguchi H, Kunicki TJ (2001) Variation in human platelet glycoprotein VI content modulates glycoprotein VIspecific prothrombinase activity. Arterioscler Thromb Vasc Biol 21: 1857-1863.

21. Perrotta PL, Perrotta CL, Snyder EL (2003) Apoptotic activity in stored human platelets. Transfusion 43: 526-535.

22. Boomgaard MN, Gouwerok CW, Palfenier CH, Pankalla-Blandeau IE Veldman HA, et al. (1995) Pooled platelet concentrates prepared by the platelet-rich-plasma method and filtered with three different filters and stored for 8 days. Vox Sang 68: 82-89.

23. Dijkstra-Tiekstra MJ, Pietersz RN, Huijgens PC (2004) Correlation between the extent of platelet activation in platelet concentrates and in vitro and in vivo parameters. Vox Sang 87: 257-263.

24. Dumont LJ, AuBuchon JP, Whitley P, Herschel LH, Johnson A, et al. (2002) Seven-day storage of single-donor platelets: recovery and survival in an autologous transfusion study. Transfusion 42: 847-854.

25. Kostelijk EH, Fijnheer R, Nieuwenhuis HK, Gouwerok CW, de Korte D (1996) Soluble P-selectin as parameter for platelet activation during storage. Thromb Haemost 76: 1086-1089.

26. Fijnheer R, Modderman PW, Veldman H, Ouwehand WH, Nieuwenhuis $\mathrm{HK}$, et al. (1990) Detection of platelet activation with monoclonal antibodies and flow cytometry. Changes during platelet storage. Transfusion 30: 20-25.

27. Badlou BA, Ijseldijk MJ, Smid WM, Akkerman JW (2005) Prolonged platelet preservation by transient metabolic suppression. Transfusion 45 : 214-222.

28. Valeri CR, Macgregor H, Barnard MR, Summaria L, Michelson AD, et al. (2004) In vitro testing of fresh and lyophilized reconstituted human and baboon platelets. Transfusion 44: 1505-1512.

29. Barnard MR, MacGregor H, Ragno G, Pivacek LE, Khuri SF, et al. (1999) Fresh, liquid-preserved, and cryopreserved platelets: adhesive surface receptors and membrane procoagulant activity. Transfusion 39: 880-888.

30. Badlou BA, Spierenburg G, Ulrichts H, Deckmyn H, Smid WM, et al. (2006) Role of glycoprotein Ibalpha in phagocytosis of platelets by macrophages. Transfusion 46: 2090-2099.

31. Josefsson EC, Gebhard HH, Stossel TP, Hartwig JH, Hoffmeister KM (2005) The macrophage alphaMbeta2 integrin alphaM lectin domain mediates the phagocytosis of chilled platelets. J Biol Chem 280: 18025-18032.

32. Badlou BA, van der Meer PF, Akkerman JW, Smid WM, Pietersz RN (2007) Metabolic energy reduction by glucose deprivation and low gas exchange preserves platelet function after $48 \mathrm{~h}$ storage at 4 degrees $\mathrm{C}$. Vox Sang 92: 311-318.

33. Leytin V, Allen DJ, Gwozdz A, Garvey B, Freedman J (2004) Role of platelet surface glycoprotein Ibalpha and P-selectin in the clearance of transfused platelet concentrates. Transfusion 44: 1487-1495.

34. Brown SB, Clarke MC, Magowan L, Sanderson H, Savill J (2000) Constitutive death of platelets leading to scavenger receptor-mediated phagocytosis. A caspase-independent cell clearance program. J Biol Chem 275: 5987-5996.

35. Hoffmeister KM, Felbinger TW, Falet H, Denis CV, Bergmeier W, et al. (2003) The clearance mechanism of chilled blood platelets. Cell 112: 87-97.

36. Jansen AJ, Josefsson EC, Rumjantseva V, Liu QP, Falet H, et al. (2012) Desialylation accelerates platelet clearance after refrigeration and initiates GPIba metalloproteinase-mediated cleavage in mice. Blood 119: 1263-1273.

37. Hoffmeister KM, Josefsson EC, Isaac NA, Clausen H, Hartwig JH, et al. (2003) Glycosylation restores survival of chilled blood platelets. Science 301: 1531-1534. 